\title{
Une centrale hydro-électrique de grande puissance et de basse chute: Ottmarsheim
}

\section{Disposition originale adoptée pour les pertuis du barrage et leur commande automatique}

\author{
Ottmarsheim : Automatic control sluices \\ PAR J.-H. LIEBER

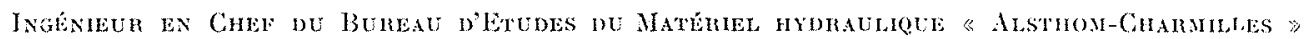 \\ English synopsis, p. 114.
}

Lorsque le grand canal d'Alsace sera termine, l'aménagement du Rhin entre Bàle et Strasbourg sera accompli du double point de vue de la navigation ot de la production hydro-clectrique.

En effet, les chalands et les péniches qui actuellement encore empruntent le lit naturel du fleuve sur la plus srande partie de ce parcours de $120 \mathrm{~km}$, quitteront le Rhin un peu à l'aval de Huningue, le port de Bâle, pour ne plus le retrouver qu'à l'entrée de Strasbourg, ayant franchi celte distance en empruntant constamment le canal d'Alsace, et traversé sept écluses pour regagner les $107 \mathrm{~m}$ de la dénivellation existante.

Au cours du siecle passé, le cours du Rhin entre ces deux villes a fait l'objet de travaux de corrections pour l'endiguer et éviter les dégâts causés par les inondations dues aux crues, mais la conséquence en fut une modification importante du fond de son lit, qui ne cesse de s'approfondir à cause de la vitesse accrue de l'eau. Des barres rocheuses sont apparues, une fois les alluvions partiellement entraînées et des rapides se sont créés, rendant la navigation dangereuse, voire impossible. C'est ainsi que, déjà maintenant, les chalands ne passeraient plus la barre d'Istein, s'ils n'évitaient cet écueil en empruntant le canal de Kembs.

Du point de vue de la production d'énergie électrique, l'équipement du Rhin sur ce tronçon présente un intérêt exceptionnel du fait de la pente moyenne relativement forte, de l'importance du débil le débil semi-permanent à Bâle est supérieur à $1.000 \mathrm{~m}^{3} / \mathrm{s}$ ) el sa rigularité due à l'importance du bassin versant $\left(36.000 \mathrm{~km}^{2} \dot{a}\right.$ Bâle) qui comprend des lacs et de grands glaciers. La production d'énergie électrique, une fois le Rhin équipé de Bâle à Strasbourgs, sera de plus de 6 milliards de $\mathrm{kWh}$.

Le premier palier du grand canal d'Alsace a été réalisé de 1928 a 1932 ; ce sont les ouvrages de Kembs qui comportent un barrage situé sur le Rhin, à $4 \mathrm{~km}$ à l'aval du port de Bâle de Huningue et un canal de force motrice et de navigation sur la rive gauche du fleuve, qui se développe sur une distance de $6 \mathrm{~km}$; à son extrémité aval, le canal se divise en deux branches, celle de gauche alimentant l'usine de force motrice, l'autre aboutissant à l'écluse, d'une dénivellation maximum de $18 \mathrm{~m}$.

L'usine de force motrice de Kembs a une production annuelle moyenne de prìs de 1 milliard de $\mathrm{kWh}$.

Le second palier du grand canal d'Alsace, Ottmarsheim, est en cours de réalisation. I.es travaux ont débuté en 1948 et sont actuellement très avancés, puisque la mise en eau des ouvrages a commencé et que la mise en route du premier groupe est prévue pour 1952.

Le canal d'amenée a environ $14 \mathrm{~km}$ de longueur; il commence directement à l'aval de l'usine de Kembs, à la naissance du canal de fuite qui, par une courbe, reliait jusqu'à maintenant Kembs au Rhin; une fois les travaux d'Ottmarsheim terminés, cette liaison dircele 
avec le Rhin sera done supprimée. Le nouveau canal d'amenée a $80 \mathrm{~m}$ de large alu plafond, $137 \mathrm{~m}$ environ de largeur au plan deau, coci sur sa plus grande étendue; ces caractéristiques sont très voisines de celles du canal de Kembs. Il est prévu pour un débit maximum de $1.160 \mathrm{~m}^{3} / \mathrm{s}$, qui peut ètre absorbé en surcharge par l'usine motrice d'Ottmarsheim. A son extrémité aval, le canal s'élargit jusqu'à avoir une largeur utile de $200 \mathrm{~m}$, ceci sur une longueur de $600 \mathrm{~m}$, créant ainsi un bassin de virage pour les péniches. Puis le canal se divise en deux branches, celle de gauche, d'une longueur de $1.200 \mathrm{~m}$ alimentant, comme à Kembs, l'usine de force motrice, celle de droite, longue de $1.000 \mathrm{~m}$ aboutissant à l'écluse qui comporte deux sas; le petit sas a $12 \mathrm{~m}$ de largeur, le grand $23 \mathrm{~m}$. Tous deux ont $185 \mathrm{~m}$ de longueur, la dénivellation maximum est de $18 \mathrm{~m}$. Des mesures spéciales ont été prises pour accroître la vitesse de remplissage et de vidange au maximum, qui est de $3 \mathrm{~m} / \mathrm{mn}$ pour le sas de $12 \mathrm{~m}$ de large et de $1,5 \mathrm{~m} / \mathrm{mn}$ pour celui de $23 \mathrm{~m}$ de large. De sorte qu'un bateau mettra 12 minutes à franchir la petite écluse et 20 minutes la grande, ce qui est un temps beaucoup plus court, toutes choses égales, que celui réalisé à l'écluse de Kembs.

L'usine de force motrice est disposée, à Ottmarsheim, perpendiculairement à la direction générale d'écoulenent de l'eau. Après de nombreuses études, et compte tenu des progrès techniques accomplis depuis la réalisation de Kembs, le débit a été réparti entre quatre lurbines qui sont toutes des Kaplan, capables d'absorber chacune à pleine charge, $270 \mathrm{~m}^{3} / \mathrm{s}$ sous toute chute comprise entre $13,40 \mathrm{~m} \mathrm{et}$ $17,40 \mathrm{~m}$. C'est donc dire que pour les chutes supérieures, la capacité de la turbine est limitée pour ne pas surcharger l'alternateur. Lor's des crues cependant, on peut surouvir la turbine et lui faire absorber $290 \mathrm{~m}^{3} / \mathrm{s}$.

Il fallait loger, sur une largeur minimum, un ouvrage comportant, d'une part les quatre chambres d'eau des turbines et d'autre part des passes de décharge capables d'évacuer à elles seules le débit total maximum du canal. La solution choisie se rapproche de celle réalisée à Kembs, mais elle en diffère sur certains points et il vaut la peine, pensons-nous, de la décrire sommairement maintenant.

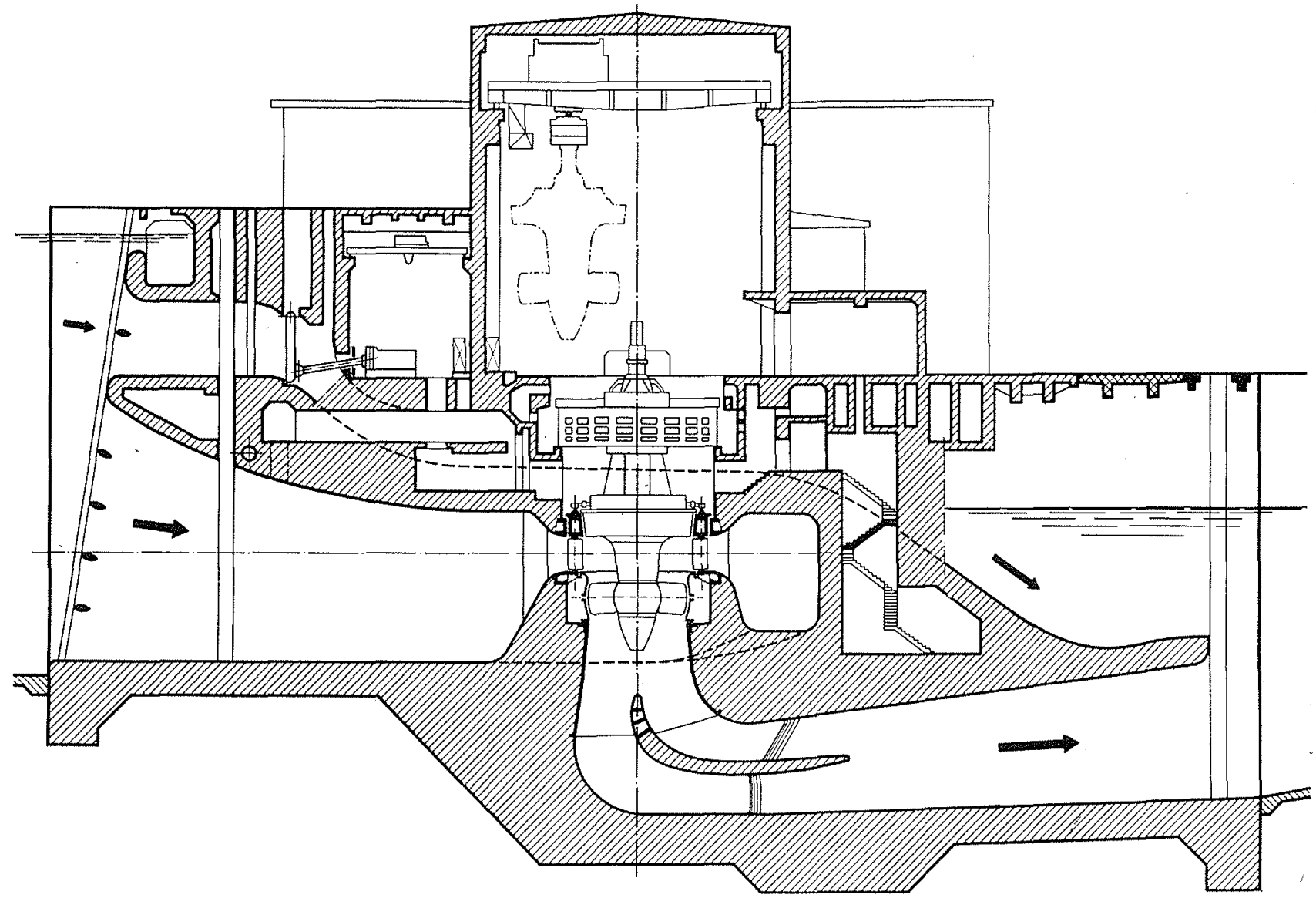

Fig. 1. - Ottmarsheim : Coupe transversale de l'usine. 


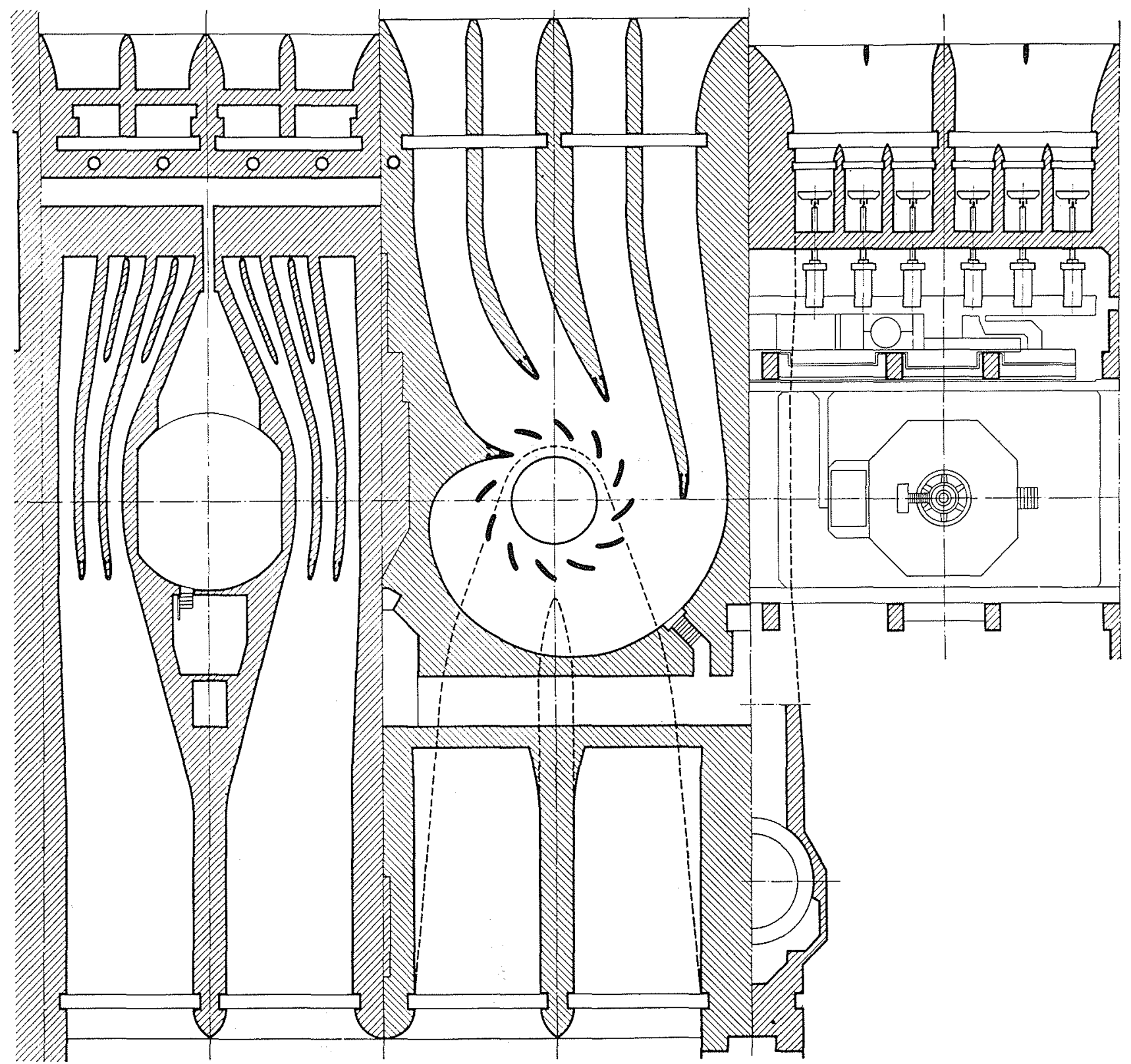

Fia, 2. - Ottmarshem: Vue en plan de l'usine.

Les quatre turbines sont alimentées par des bâches spirales en béton de forme tout à fait classique et l'eau est évacuée à l'aval par des aspirateurs coudés avec languette (fig. 1). Soit dit en passant, ces ouvrages sont de dimensions imposantes, puisque, à la grille, la prise d'eau d'une turbine mesure $24,5 \mathrm{~m}$ de largeur sur $16 \mathrm{~m}$ de hauteur environ, et que la section de sortie de l'aspirateur mesure $20,5 \mathrm{~m}$ de largeur sur $8 \mathrm{~m}$ de hauteur. La distance entre le pied de la grille de la prise d'eau et la sortie de l'aspirateur est de $75 \mathrm{~m}$. Juste à l'amont, au- dessus des chambres d'eau, sont disposées les bouchures des passes de décharge, qui sont au nombre de deux par groupe. Elles sont complètement immergées et les déchargeurs sont donc en charge; une grille mobile en protège l'entrée. Les passes de décharge traversent de part en part l'usine et débouchent à l'aval sur un coursier incliné disposé sur le plafond des aspirateurs. Les deux passes de décharge intéressant une turbine, ont tout d'abord un tracé parallèle, vu en plan, puis se séparent pour contourner le puits en béton armé dans lequel est disposé l'al- 


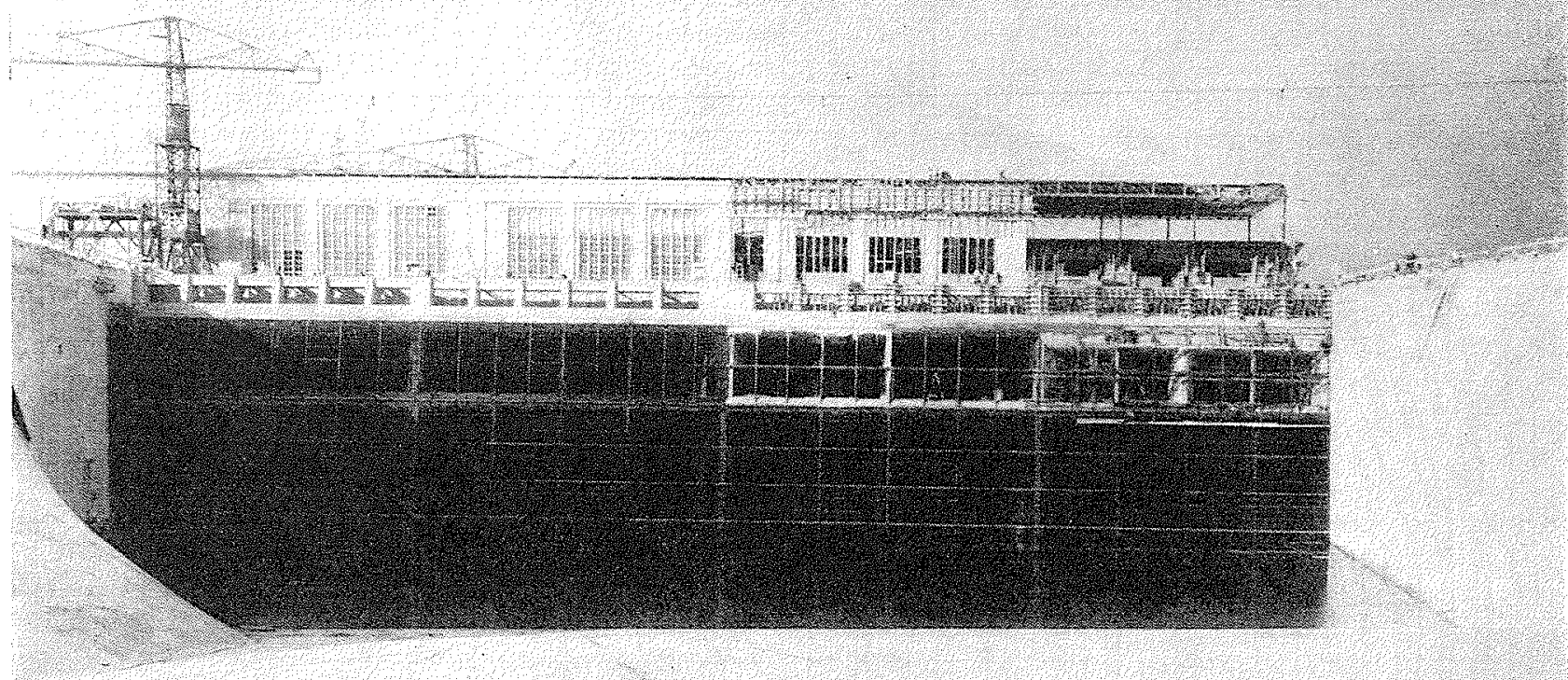

Flci. 3. - Otrianshem : Fagade amont de lusine.

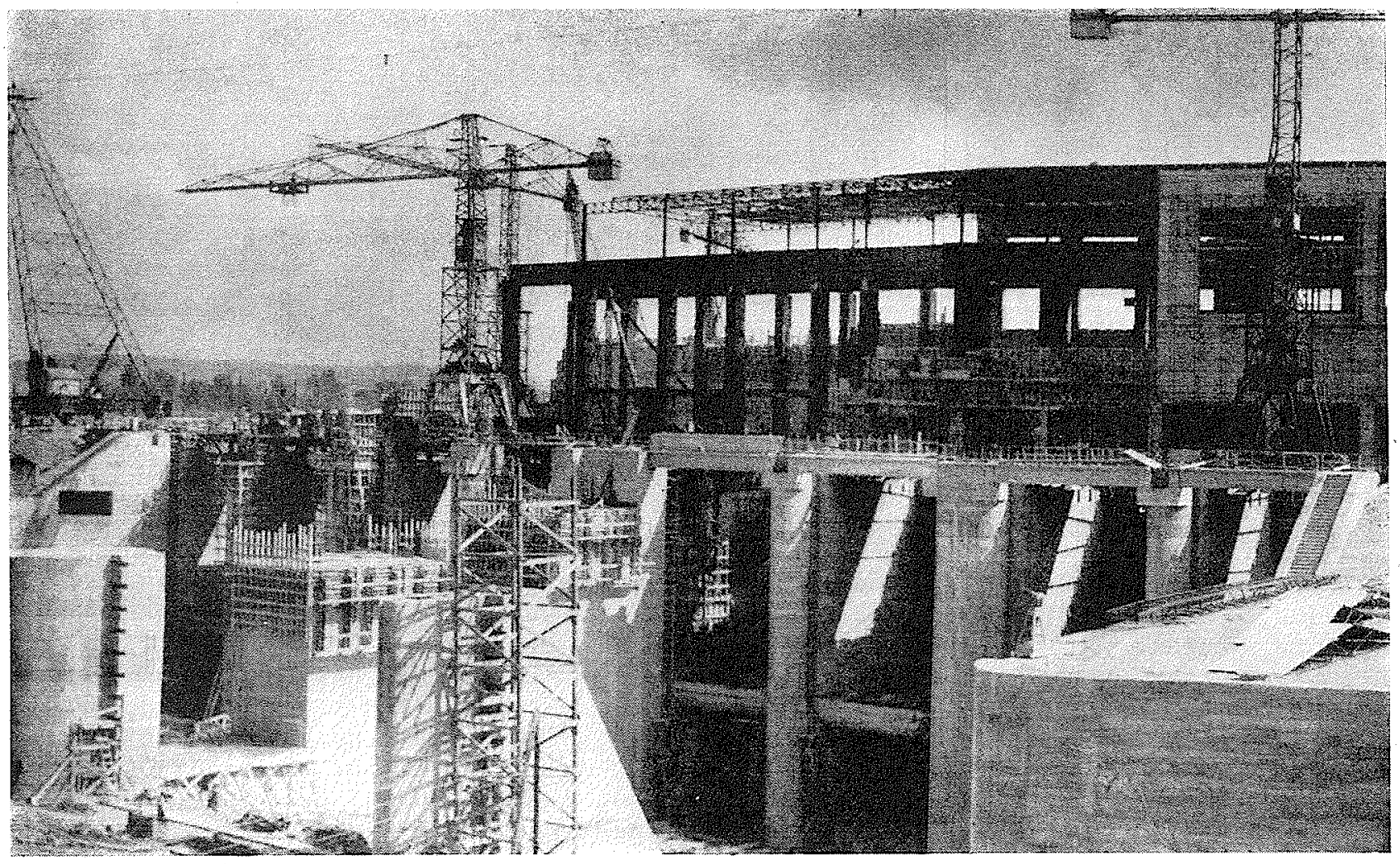

Fiti, 4. -- Otrmarshem: Ensemble du chantier de l'usine vu de l'aval. 


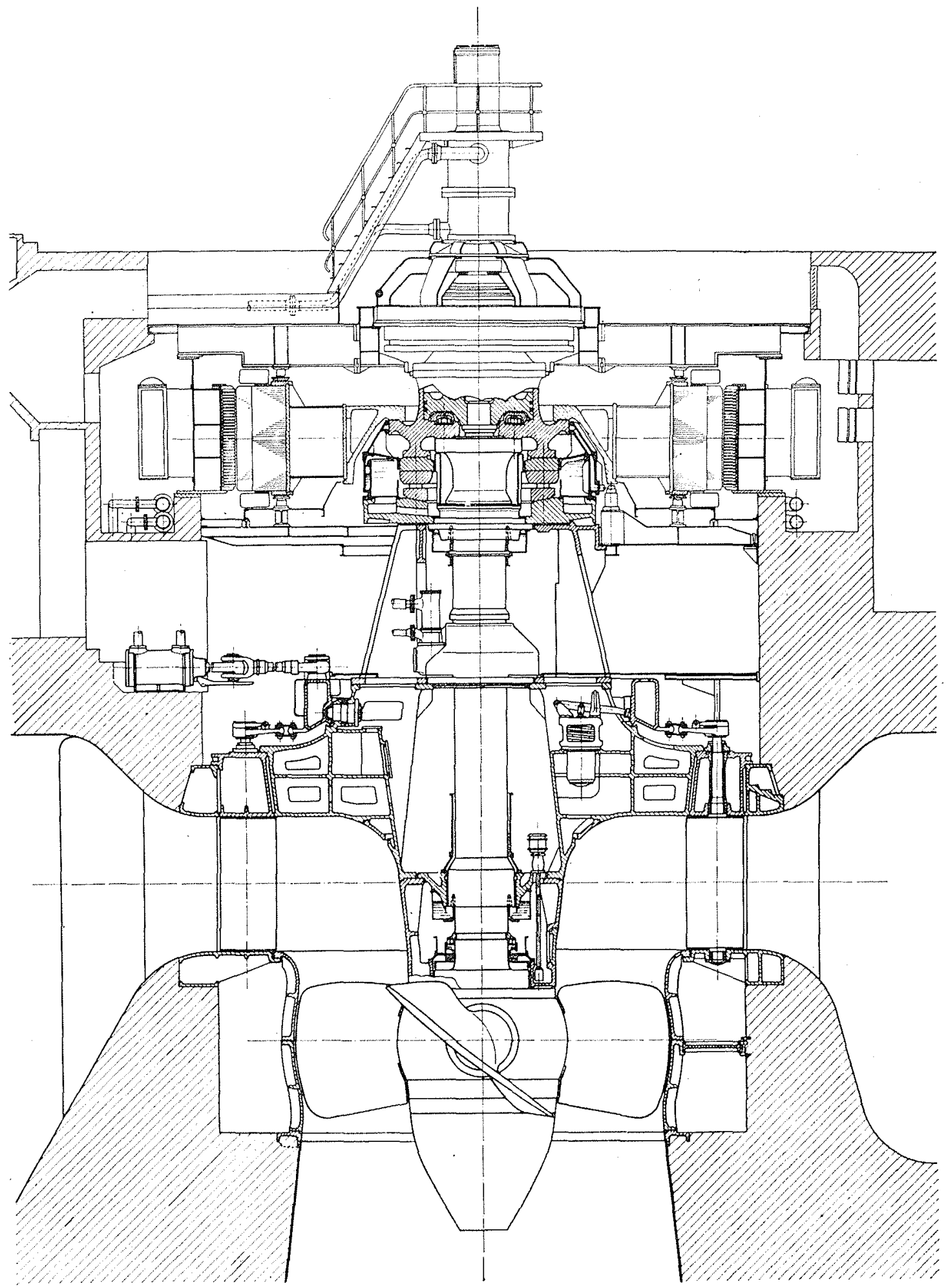

Fig. 5. - Ottmarshem : Coupe d'un groupe de $44.000 \mathrm{kVA}$.

ternateur pour se rejoindre à nouveau à l'aval de la salle des machines (fig. 2). Chaque passe est divisée, à $8 \mathrm{~m}$ environ de sa bouchure amont, en trois pertuis par des pilettes de béton armé qui se poursuivent jusqu'un peu à l'aval de l'axe longitudinal de la salle deş machines et servent de guideaux.

Chacun de ces pertuis est obturé par un clapet de fond à commande par huile sous pression. Il y a done vingt-quatre clapets pour l'en- 


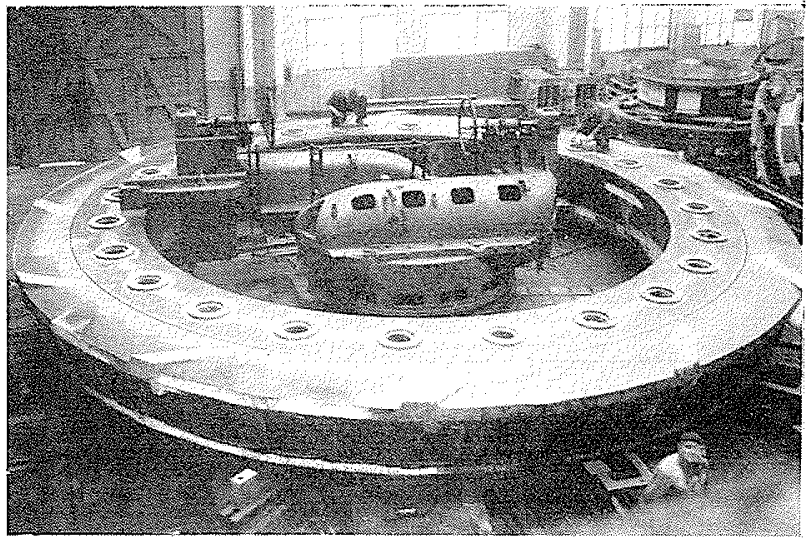

Fig, 6. - Otrmarshem.

Fond annulaire et couronne supéricure de l'anneau d'entretoises (diamètre extéricur : $10 \mathrm{~m}$, poids: 96 tomnes).

semble de l'usine et nous reviendrons plus loin sur leurs caractéristiques. Il suffira, pour l'instant, de noter qu'ils fonctionnent par tout ou rien, et que l'écoulement à l'aval de ceux-ci est à veine dénoyée; un puits de large dimension prévu à l'aval des clapets assure la ventilation. Des batardeaux sont naturellement prévus à l'amont, permettant de les isoler chacun séparément. Etant donné les servitudes imposées par la présence des groupes et de leurs accessoires et le débit important devant passer par chaque clapet, qui est de $60 \mathrm{~m}^{3} / \mathrm{s}$ pour la cote normale de 230 du plan d'eau amont, la place est très exiguë, et ce n'est pas sans peine qu'un tracé satisfaisant des canaux a été obtenu après exécution d'essais très complets sur modèle réduit par les Services du Laboratoire d'Hydraulique de Chatou, rattaché au Service des Etudes et Recherches d'Electricité de France. Il a fallu, notamment, prévoir un guideau supplémentaire à l'aval de chaque clapet et jouer sur les congés à grand rayon dans les coins de la section rectangulaire des pertuis pour éviter que la nappe d'eau s'écoulant sous les clapets, vienne coller au plafond d'une part, et crée des affouillements à l'aval d'autre part. (Voir fig. 3 et 4 .)

La salle des machines a $20,5 \mathrm{~m}$ de hauteur sous plafond, $18 \mathrm{~m}$ de large et $102 \mathrm{~m}$ de long, plus un hall de montage de $20 \mathrm{~m}$ et un hall de décuvage de $23,50 \mathrm{~m}$ en alignement; seuls les excitatrices et les chapeaux de réglage des roues Kaplan sont apparents, les tòles de couverture des alternateurs étant même encastrées dans des puits de 1,20 in de profondeur.

Chaque groupe, constitué par une turbine Kaplan de $53.000 \mathrm{ch}$ entrainant un alternateur triphasé de $44.000 \mathrm{kVA}$ sous $10.000 \mathrm{~V}$ à la vitesse de $93 \mathrm{t} / \mathrm{mn}$, est monobloc (fig. 5). Il y a une liaison intime entre la turbine et l'alternateur; un arbre unique lie la roue Kaplan au rotor de l'al- ternateur, arbrc guidé par deux paliers seulement, l'un sur la turbine, lautre sous l'alternateur, ce dernier combiné d'ailleurs avec le pivot de 1.100 tonnes qui supporte toutes les charges mécanicutes des parties tournantes el les charges hydrauliques. Ce pivot est porte directement par le fond de la turbine, qui transmet toutes ces charges directement aux fondations, par l'intermédiaire de pieces métalliques et mécaniques, sans interposition du classique plancher en béton supportanl l'alternateur. A Othmarsheim, le stator repose sur un puits en béton armé de tris faible hauteur, entourant la turbine. Autro détail constructif qui illustre bien ce concept do bloc turbine-alternateur : la pièce combinée qui coilfe l'arbre du groupe et qui sert en mème temps de manchon d'entrainement du tourteau du rotor, de cylindre du servo-moteur actionnant la roue Kaplan et de support de l'anneau glissant du pivot. Cette pièce, extrèmement importante, est l'une des plus difficiles à róaliser pour le métallurgiste, par sa forme et ses dimensions (diamètre de l'alésage : $1.800 \mathrm{~mm}$ ) et son poids (environ 30 t).

Le groupe, ainsi constitué, est extrêmement ramassé, ce qui est un avantage évident au point de vue mécanique; il n'y a plus, à proprement parler, d'étage turbine, mais, sous l'alternateur, un puits d'accès sur le fond de la turbine juste assez haut pour une circulation aisé du personnel de service. On peut dire qu'une centrale de ce type est à un seul itage: celui de la salle des machines, siluć au-dessus des alternateurs. (Voir fig. 6 et 7 .)

Il ne faut pas oublier que Ottmarsheim est le deuxième chainon sur le grand canal d'Alsace, Kembs étant la première usine sur celuici. Pour des raisons d'exploitation, il convenait de maintenir le débit constant à l'aval de l'usine

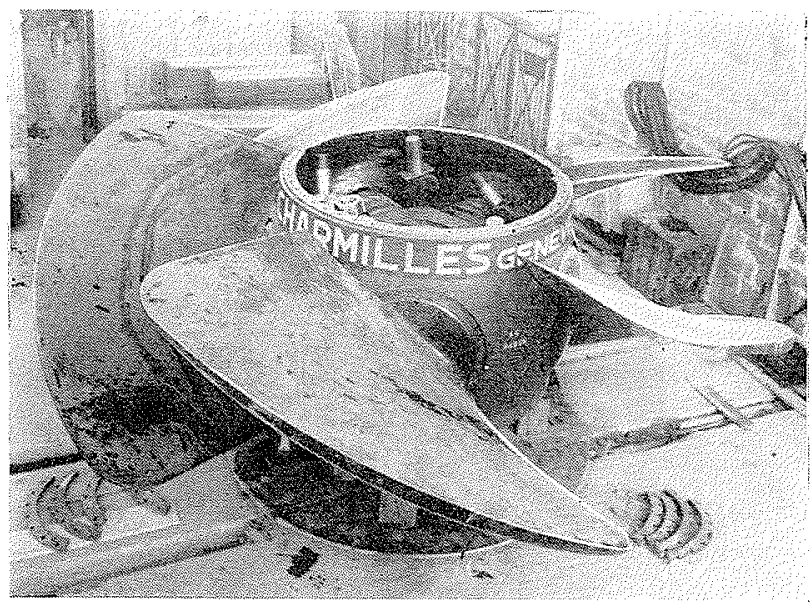

Fig. 7. - Otrmarsheim.

Roue d'une turbine Kaplan de $53.000 \mathrm{ch}$ 
d'Ottmarsheim en toutes circonstances, quelle que soit la charge imposée à l'usine; de plus, il fallait éviter des fluctuations brutales du débit dans le canal pour ne pas gêner la navigation qui, ainsi que nous l'avons vu plus haut, emprunte celui-ci. C'est pourquoi un certain automatisme fut recherché pour la commande des vannes des passes de décharge d'Ottmarsheim.

Les principes directeurs que nous avons suivis pour réaliser cet équipement sont les suivants :

$1^{\circ}$ Il faut assurer dans le temps la constance du débit de consigne s'écoulant au travers des ouvrages d'Ottmarsheim dans le grand canal d'Alsace.

2" L'incident imprévisible pouvant amener une perturbation brutale dans la vaTeur de ce débit de consigne est la fermeture rapide d'une ou plusieurs turbines, consécutive à une disjonction des alternateurs du réseau de transport d'énergie, pour une raison quelconque. On s'attachera, dès lors, à ce que toute fermeture notable du vannage d'une turbine entraîne automatiquement l'ouverture d'une ou plusieurs vannes pour compenser sensiblement la diminution du débit s'écoulant au travers des turbines par une augmentation du débit s'écoulant par les passes de décharge. Les calculs ont montré qu'en cas de disjonction totale des quatre groupes d'Ottmarsheim fonctionnant en surcharge avec un débit de $1.160 \mathrm{~m}^{3} / \mathrm{s}$ dans le canal d'amenée, et si l'on n'ouvre aucune passe de décharge, le plan d'eau amont subit alors une montée de $50 \mathrm{~cm}$ en $31 / 2 \mathrm{mn}$. Le temps dont on dispose pour réagir est done très court; de plus il faut tenir compte de l'onde provoquée par la fermeture brusque des turbines et dont l'amplitude est de l'ordre de grandeur de $1 \mathrm{~m}$; or la revanche des digues à l'amont n'est que de $1,5 \mathrm{~m}$ el la sécurité est done bien modeste ${ }^{1}$.

$3^{\circ}$ Les mouvements d'ouverture des vannages des turbines nécessités par des prises de charge des alternateur's selon un programme préalable, s'effectuent lentement et sont contrôlés depuis le poste de commande de la centrale; la refermeture

1. Pour vérifier les caleuls préalables qui avaient été faits, Electricité de France a fait des mesures à Kembs. Par la suite, des calculs plus précis ont été faits pour Ottmarsheim et ont donné les résultats suivants : en $3 \mathrm{mn} 7 \mathrm{~s}$, temps pris par l'onde pour parcourir le canal de force motrice seul, de $1.662 \mathrm{~m}$ de longueur (de la grille d'entrée des turbines jusqu'ì la jonction avec le canal de navigation en remontant vers l'amont), la montée du plan d'eau moyen est au total, devant les grilles d'entréc des turbines, de $1,53 \mathrm{~m}$.

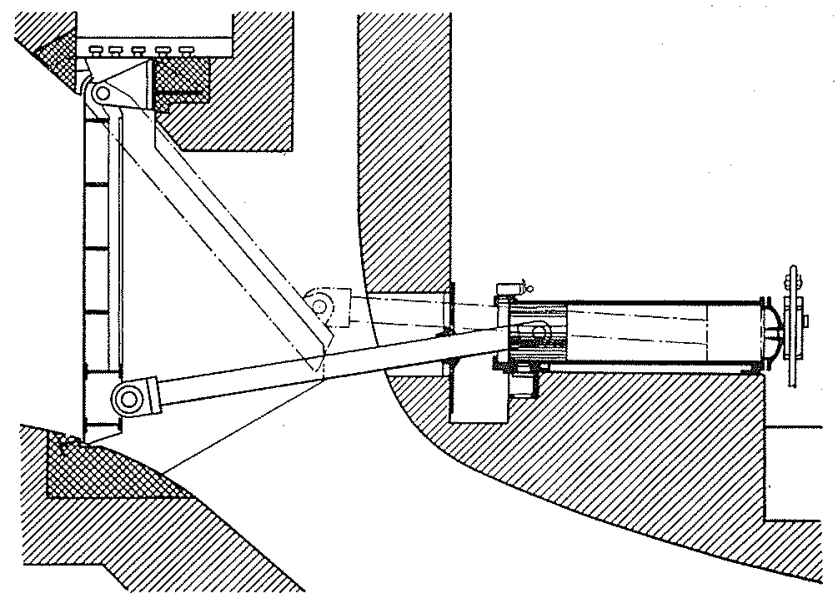

Fic. 8. - OtTmanshenr.

Coupe d'un clapet et de son servo-moteur de commande.

des vamnes pour rétablir la constance du débit se fera également sous Te contrôle du personnel de la salle de commande et non automatiquement.

$4^{\circ}$ On doit pouvoir choisir à volonté les vannes commandées à l'ouverture par l'une des turbines.

$5^{\circ}$ Etant donné l'importance attachée au maintien du débit de consigne, il est capital de pouvoir contrôler constamment du poste de commande l'état et le fonctionnement des vannes; toutes les opérations sélectives se feront dès lors des tableaux des vannes, groupés au poste de commande de la centrale, d'où l'on aura, de plus, la possibilité de déclancher manuellement aussi bien l'ouverture que la fermeture d'une vanne quelconque.

Pour obturer les vingt-quatre pertuis des passes de décharge, on a choisi finalement des clapets de fond à cause de leur simplicité, de leur grande sécurité de fonctionnement et parce qu'ils s'ouvrent simplement sous l'action de la poussée de l'eau.

Des essais sur modèle réduit à l'air et à l'eau furent entrepris par le constructeur pour vérifier les lois de poussée et de débit. Ces essais permirent de vérifier que le débit de $60 \mathrm{~m}^{3} / \mathrm{s}$ pourrait bien être évacué par un clapet, la cote du plan d'eau amont étant de 230.

Chaque clapet est constitué par un panneau en tôle d'acier convenablement nervuré, de $2.800 \mathrm{~mm}$ de large sur $3.800 \mathrm{~mm}$ de hauteur, suspendu à deux paliers supérieurs, autour desquels il pivote (fig. 8). Les paliers sont fixés à une poutre support solidement ancrée dans le béton. Des blindages latéraux en tôle au chrome aluminium peu oxydable, sont scellés dans les parois latérales des pertuis au moyen d'un dis- 
positif spécial permettant de les dresser convenablement. Le clapet est muni d'étanchéités sur ses quatre còtés, un seuil garni de caoutchouc spécial est scellé dans le sol du pertuis, un blindage en arc de cercle, contre lequel s'appuic un cuir d'étanchéité est scellé au plafond. Une bielle d'acier attaque le panneau du clapet au milieu de sa poutre inférieure, par l'intermédiaire d'une rotule en bronze, ceci pour éviter une déformation de l'attache si un corps étranger venait se coincer dans un angle du clapet lors d'une fermeture. Les blindages latéraux et la bielle sont tous munis de corps de chauffe électriques puissants, ceci pour ćviter le givrage en période de gel et assurer un fonctionnement correct des clapets, même en hiver. A son autre extrémité, la bielle est fixée au piston du servomoteur à huile de commande, à simple effet. En mettant le cylindre sous pression, on ferme le clapet en eau vire. En mettant le cylindre à l'échappement, le clapet s'ouvre, ce mouvement étant contrôlé et freiné en fin d'ouverture en diaphragmant l'évacuation de l'huile. Le clapel fonctionne par tout ou rien; il s'ouvre en 15 secondes et se referme en un temps notablement plus long.

Les vingt-quatre cylindres de commande des clapets sont disposés avec tous les accessoires nécessaires à leur fonctionnement dans une salle de commande des clapets altenante à la salle des alternateurs et au mème niveau. Un masque d'étanchéité évite les projections d'eau dans la salle à l'endroit où la bielle traverse le mur amont.

Trois clapets sont alimentés en huile sous pression à $15 \mathrm{~kg}$ par une même station de pompage; il $y$ en a done huit au total, comportant chacune une clectropompe à gros débit qui n'entre en service que pour les manœuvres de refermeture, une électro-pompe à faible débit qui fonctionne en permanence pour compenser les fuites des organes de réglage et un aceumulateur à huile sous pression d’air qui permet de maintenir les clapets fermés pendant un certain temps en cas d'arrêt intempestif des pompes, laissant ainsi au personnel le temps d'intervenir utilement. Ia mise en et hors action de la grosse pompe est commandée automatiquement par un manostat. En cas de manque de pression d'huile, et pour éviter l'ouverture intempestive d'un clapet, on peut toujours le verrouiller en position de fermeture. Le déverrouillage, qui ne peut s'effectuer que par pression d'huile, pent encore se faire cependant en cas de nécessité absolue, en utilisant une petite électro-pompe à courant continu alimentée par la batterie, el prévue à cel usage; ceci permet alors d'ouvrir le clapel incriminé en cas d'urgence, par exemple si les groupes disjonctent alors qu'il y a panne de courant alternatif sur les auxiliaires généraux de la centrale.

La manœuvre d'ouverture et de fermeture d'un clapet est provoquée en actionnant à distance l'électro-aimant d'ouverture ou l'électroaimant de refermeture du relais pilote agissant sur le distributeur du servo-moteur du clapet.

Comme nous l'avons déjà signalé, les turbines peuvent absorber en surcharge $1.160 \mathrm{~m}^{3} / \mathrm{s}$; chaque clapet débitant $60 \mathrm{~m}^{3} / \mathrm{s}$ en position d'ouverture, il faut ouvrir 20 clapets pour laisser passer sùrement un débit équivalent.

A un autre point de vue, on conçoit qu'on peut régler le débit total maximum pouvant s'écouler dans les ouvrages a $60 \mathrm{~m}^{3}$ prís, e'està-dire en gros à $1 / 20^{\circ}$ près, ce qui montre la souplesse du systime. En réalité, on peut faire encore mieux pratiquement, car tout débit à régler peut être encadré par excès ou par défaut en jourant sur l'ouverture ou la fermeture d'un clapet et le débit est donc ajusté avec une précision de quelques $\%$ du débit maximum du canal; l'erreur est alor's négligeable, car les variations de niveau qui en résultent seront très lentes, sensibles seulement après quelques heures, et le chef de quart pourra les contrôler par l'observation des limnigraphes du poste de commande et intervenir alors en ouvrant ou en refermant un clapet.

A toute époque de l'annce, on peut isoler, visiter, réparer au moins quatre clapets, ou au moins un pertuis de décharge.

Si un groupe turbine-alternateur est en révision, on met sa turbine entre batardeaux; il est alors préférable de ne pas utiliser les clapets qui le surmontent. Il reste alors 18 clapets utilisables qui peuvent ensemble évacuer $1.080 \mathrm{~m}^{3} / \mathrm{s}$; or le débit du canal ne dépasse cette valeur que durant cinq mois par année. On peut donc durant sept mois isoler un groupe entre batardeaux.

On peut isoler deux groupes entre batardeaux tant que le débil du canal reste inférieur à $720 \mathrm{~m}^{3} / \mathrm{s}$, soit durant environ trois mois et demi par an.

On voit donc que l'on dispose d'un temps raisonnable au cours de l'année pour les travaux d'entretien sur les groupes et les clapets, car il a été admis qu'on ne mettrait jamais plus de deux groupes derrière batardeaux simultanément.

Un groupe fonctionnant en surcharge et absorbant $290 \mathrm{~m}^{3} / \mathrm{s}$, il faut ouvrir cinq clapets s'il disjoncte pour rétablir la constance du débit à l'aval. Le cercle de vannage de la turbine actionne au cours de son mouvement de rotation et successivement, cinq contacts; chacun d'eux peut servir à commander le déclenchement de la manouvre d'ouverture d'un clapet en agis- 
sant sur son électro-aimant d'ouverture. Le principe de la conjugaison est done extrêmement simple. Encore fallait-il imaginer un dispositif de contrôle capable d'éviter toute fausse manœuvre; en effet, il fallait pouvoir actíonner avec un quelconque des cinq contacts d'une turbine, l'un quelconque des 24 clapets de décharge, et disposer d'un verrouillage absolument sûr évitant de façon certaine qu'un mème clapet puisse être commandé par deux contacts différents, ou que, récioroquement, deux clapets puissent être commandés simultanément par un seul et même contact de turbine.

Tous les appareils de contrôle et de commande des clapets ont été groupés, au poste de commande, sur cinq tableaux qui occupent la place centrale du poste et sont sous les yeux de l'homme de quart.

Le panneau central comporte des indicateurs de niveaux; étant donné l'importance que l'on attache à Ottmarsheim à la constance de la cote du niveau amont et du niveau aval en exploitation, il est nécessaire que l'homme de quart responsable de la centrale puisse à chaque instant vérifier ces niveaux. Il est done prévu un limnigraphe enregistreur pour le niveau amont et pour le niveau aval; un limnimètre différentiel indique à chaque instant la chute disponible. La place de ces instruments sur le panneau central des clapets est tout indiquée; en effet, si, par exemple, le niveau aval baisse, c'est que trop peu d'eau s'écoule du barrage d'Ottmarsheim pour maintenir la cote de consigne et qu'il faut ouvrir des clapets supplémentaires; si le niveau amont monte, risquant de submerger les berges, il faut aussi ouvrir de nouveaux clapets et ainsi de suite.

La partie inférieure de ce panneau est occupée par le tableau de présélection. C'est un tableau de fiches à combinaisons multiples qui permet d'établir la liaison électrique entre chaque contact de vannage des turbines et l'électro-aimant de déclenchement à l'ouverture de chaque clapet. Le tableau de présélection est conçu de telle façon qu'un contact de vannage donné ne peut jamais commander qu'un seul clapet, mais également un clapet déterminé ne peut en aucune façon être commandé par deux contacts différents; il n'y a donc aucune fausse mancuvre possible.

La face avant du tableau se présente sous la forme d'une porte; sur cette porte sont fixées quatre plaques de couleurs différentes, rouge, vert, jaune, bleu à la partie supérieure, et en regard, quatre plaques de même couleur, à la partie inférieure (fig. 9). Les plaques supéricures qui schématisent les quatre turbines, comportent chacune cing voyants transparents, qui sont les cinq contacts de vannage. Les plaques inférieures comportent deux rangées de

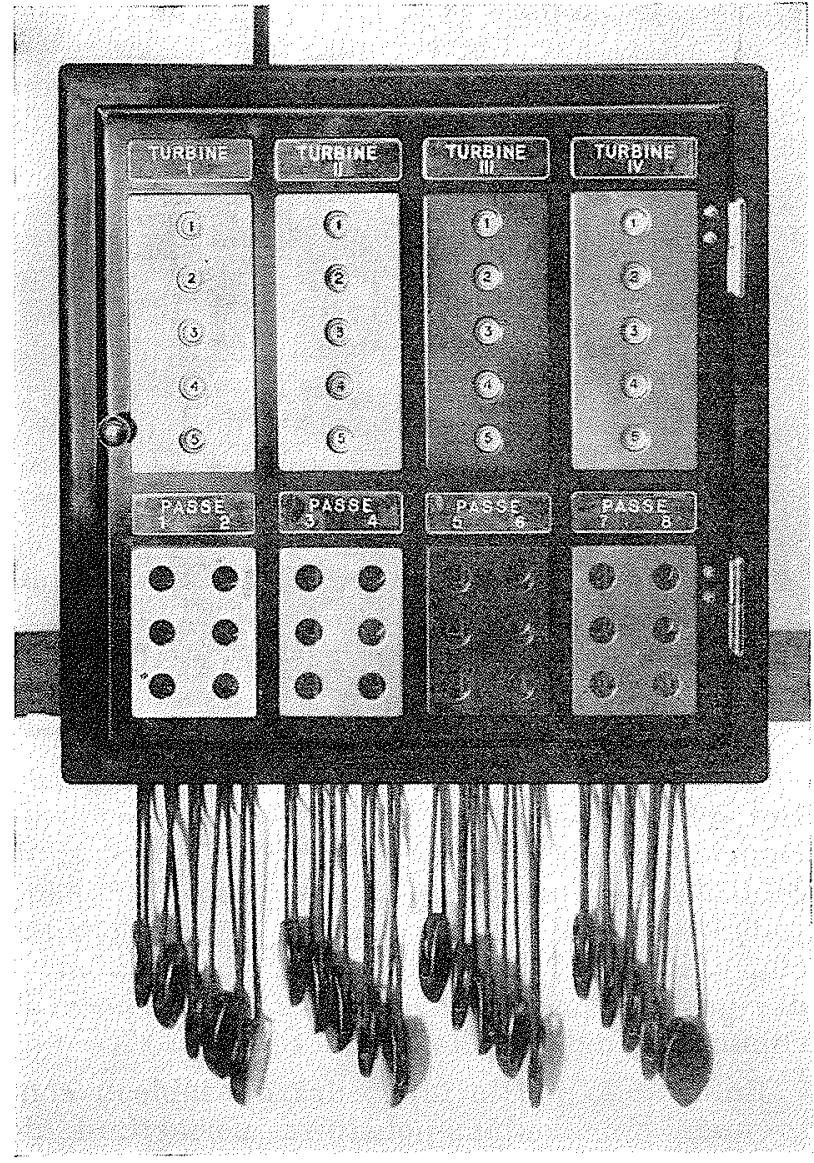

Fig. 9. - Ottmarshem.

Le tableau de présélection de la commande des clapets, porte fermée.

trois voyants transparents et représentent les deux passes de trois elapets de chaque turbine.

Lorsqu'on ouvre la porte, on voit alors les vingt fiches spéciales terminant les cordons souples reliés aux vingt contacts de vannage des turbines, enfoncées dans les jacks de repos de la partie supérieure du coffret; chaque fiche est recouverte sur sa face frontale par une pastille de la couleur de sa turbine (rouge, vert, jaune, bleu) et du numéro d'ordre de son contact (1 2-3-4-5). En prenant une fiche et en l'enfoncant dans l'un des 24 jacks actifs de la partie inférieure du coffret, on «prépare» le clapet dont on a choisi le jack. Une fois la porte du tableau de présélection refermée, on peut d'un seul coup d'œil de l'extérieur :

$1^{-}$Voir quels sont les contacts des turbines non utilisés: en effet derrière les voyants transparents des plaques «turbines», les têtes de fiches apparaissent avec leur couleur et leur numéro d'ordre. 
2" Voir le nombre de clapets "préparés»; en effet, par les voyants transparents des plaques «clapets», apparaissent les têtes des fiches utilisées enfoncées dans les jacks.

3" Connaitre quel est l'emplacement des clapets "préparés» d'une part, el sous la dépendance de quelle turbine d'autre part, chaque clapel est placé. En effet, la couleur de la tête de la fiche visible par le voyant transparent renseigne immédiatement l'observateux.

Le fait d'enfoncer une fiche dans un jack de clapet entraine la fermeture d'un contact addilionnel de signalisation, donnant l'indication lumineuse de clapet préparé sur l'un des panneaux latéraux.

De part et d'autre du panneau central sont montés deux panneaux, soit quatre en tout. Chacun d'eux se rapporte à deux groupes de trois clapets, intéressant une turbine, et leurs deux centrales de pompage. Des voyants lumineux judicieusement groupés indiquent pour chaque clapet son état, de mème que le fonctionnement des groupes de pompage et des mécanismes de commande des clapets.

Enfin, à la partie inférieure du tableau sont groupés six boutons-poussoirs permettant de commander en toul temps l'ouverture des clapets correspondants, a condition cependant qu'ils ne soient pas verrouillés, et les six bouions-poussoirs permettant de déclencher la refermeture des clapets, pour autant que l'on dispose de pression d'huile.

Un bouton-poussoir général de verrouillage est prévu par groupe de six elapets, bouton sur lequel il faut appuyer en même temps que sur celui d'ouverture, pour rendre effectif l'ordre d'ouverture manuelle, ceci pour éviter toute fausse manœuvre ayant pour conséquence l'ouverture intempestive d'un clapet.

Un panneau de signalisation situé au tableau de groupe donne à peu près les mèmes indications a l'homme de quart aux machines, mais ce dernier ne peut pas commander les clapets ni à l'ouverture ni à la fermeture. En cas de besoin, cependant, ces manœuvres sont possibles en agissant manuellement directement sur le relais pilote du tiroir du servo-moteur des clapets.

Nous voyons donc qu'avec des moyens très simples, on a rempli les conditions de base imposées, tout en évilant de tomber dans l'excès d'un automatisme complet qui aurait imposé la nécessité d'utiliser un appareillage électrique compliqué et délicat. Bien an contraire, nous avons cherché à faciliter la tâche de l'homme de quart du poste de commande tout en lui laissant la possibilité de se rendre compte à chaque instant et aisément de l'état de préparation de sa centrale, el l'initiative d'intervenir pour redresser un déséquilibre dans le débit passant au travers des ouvrages. C'est lui qui fait ses prévisions quant au nombre de clapets à preparer, mais c'est la machine qui intervient seule, lorsque la disjonction imprévisible survient. L'avenir proche nous dira si pratiquement nos prévisions se réalisent, et si les dispositifs adoptés sont efficaces.

Avec ses $176.000 \mathrm{kVA}$ installés, Ottmarsheim, deuxième chaìnon du grand canal d'Alsace, ajoutera son milliard de kWh annuellement produits au milliard de $\mathrm{kWh}$ que Kembs déverse déjà sur le réseau français.

\section{I S C USS I O N}

M. Tovnasse remalque que le nombre des clapets prévus, d'ailleurs supérieur au nombre nécessaire pour la décharge totale, aurait pu être encore inférieur à ce dernier si l'on avait tenu compte du débit des turbines marchant à vide.

M. Lrener répond que quelques clapets ont eté prévus en plus du nombre nécessaire pour parer à l'éventualite de réfection, réparation ou révision; que, d'autre part, on aurait pu tenir compte du débit de marche à vide, qui est de l'ordre de $50 \mathrm{~m} * / \mathrm{s}$ par turbine, mais qu'il n'est pas toujours possible de compter sur ce débit, notamment lors de eertains incidents, tels que defaut d'alimentation des auxiliaires, dans lesquels les machines disjonctées ne retombent pas en marche à vide mais ferment complètement.

M. Tourasse demande, d'autre part, des précisions sur le fonctionnement des dechargeurs en cas de variation de débit.

M. Lieber précise qu'à chaque changement intervenant dans la charge de la turbine selon le programme fixé à l'usine, correspond un changement d'état dans la préparation des clapets de décharge, et que d'autre part, les contacts de vannage qui agissent sur l'ouverture ou la fermeture d'un clapet préparé sont a fourchette afin qu'ils ne réagissent pas aux oscillations de réglage.

M. le Président remercie M. Lŕmer.

La séance est levée. 